\title{
Demonstration of the wide control range $Q$ factor of ring cavity with ultrashort directional coupler and curved photonic-crystal ring waveguide
}

\author{
Jun-ichiro Sugisaka, ${ }^{1,2, *}$ Noritsugu Yamamoto, ${ }^{1}$ Makoto Okano, ${ }^{1}$ Kazuhiro Komori, ${ }^{1}$ \\ and Masahide Itoh $^{2}$ \\ ${ }^{1}$ Photonics Research Institute, National Institute of Advanced Industrial Science and Technology, \\ 1-1-1 Umezono, Tsukuba, Ibaraki, Japan \\ ${ }^{2}$ Applied Physics Laboratory, University of Tsukuba, 1-1-1 Tennoudai, Tsukuba, Ibaraki, Japan \\ *Corresponding author: jun-ichiro.sugisaka@aist.go.jp
}

Received January 11, 2012; revised April 11, 2012; accepted April 20, 2012; posted April 25, 2012 (Doc. ID 161361); published June 1, 2012

\begin{abstract}
We extend the control range of the $Q$ factor of a ring cavity that consists of a photonic crystal. The control range, which determines the storage time (high $Q$ ) and efficiency of input and output (low $Q$ ) of light, is required to be wide for the random-access memory of an optical pulse train. The conventional photonic-crystal ring cavity with a directional coupler and a hexagonal-shape ring waveguide has a very narrow range. We replace these components by a directional coupler having flat dispersion and a circularly curved ring waveguide. We experimentally varied the $Q$ factor by thermal modulation of the device and achieved a control range between $1.9 \times 10^{3}$ and $1.7 \times 10^{4}$. (C) 2012 Optical Society of America

OCIS codes: $\quad 050.5298,210.4680$.
\end{abstract}

\section{INTRODUCTION}

In optical memory, a device to delay or buffer optical pulse trains is essential to achieve optical packet switching. In recent years, various approaches have been proposed to randomly access an optical pulse train, including nanocavities [1] and optical fiber loops [2]. Optical fiber loops have superior flexibility in the number of optical pulses that can be stored. However, they are difficult to miniaturize and integrate because they require a long fiber loop. To overcome this problem, we focus on the optical properties of photonic crystals. A photonic crystal $[3,4]$ is an optical material that has a periodic refractive index distribution. Such materials have unique band structures for the photon, such as bandgaps, and are used in a variety of applications for optical devices. For example, a line defect in a photonic crystal enables the incident light to confine strongly, owing to the photonic bandgap. Until now, a variety of structures having a waveguide loop (called a ring waveguide) have been integrated on semiconductor wafers by a dielectric planar waveguide circuit [-ㅡㄹ, a two-dimensional photonic crystal [9-11], a photoniccrystal waveguide bend and beam splitter [12], and a ring waveguide with periodic defects (a one-dimensional photonic crystal) [13]. These structures have been used in lasers $\underline{9}$ and wavelength filters $[\underline{5}-\underline{8}, \underline{11}]$. Moreover, the defect optical waveguide has a low group velocity frequency band, which realizes storage of optical pulses for a long time in a small area. Until now, for the purpose of development of a miniaturized buffer memory integrated on a semiconductor slab, we have designed and fabricated a microring cavity [14 $\underline{15}]$. For use as a buffer memory, the ring cavity is required to switch between two states: a reading or writing $(\mathrm{R} / \mathrm{W})$ state that can be input or output optical pulses and a storage state that can store the optical pulses in the ring waveguide. That is achieved by dynamically controlling the $Q$ factor, which indicates the strength of light confinement. So far, however, dynamic control of the ring cavity has not been demonstrated, and the dynamic characteristics have not yet been understood.

In this study, first, we discuss the available control range of the $Q$ factor for the conventional ring cavity to show that a large control range can hardly be obtained. Second, we redesign the cavity structure for a wide control range of the $Q$ factor. The fabricated device is dynamically controlled by the state of the optical switch in the ring cavity and demonstrates the controlling of the $Q$ factor. After that, we evaluated the dynamic characteristics of the ring cavity from the obtained control range of the $Q$ factor.

In Section 2 , we describe the principle of buffer memory. In Section 3 , we express the states of the memory from the aspect of $\bar{Q}$ factors to show the method of state switching. We also discuss the expected control range of the $Q$ factor for the conventional ring cavity in that section. The fabricated device, especially its replaced components, is described in Section $\underline{4}$, which is followed by the experimental results and discussion.

\section{PRINCIPLE OF RING BUFFER MEMORY}

A ring buffer memory consists of a photonic-crystal directional coupler optical switch and a ring waveguide, as shown in Fig. 1(a). It has an input/output (I/O) waveguide and a ring waveguide, and they optically couple via a directional coupler. A directional coupler is a pair of two parallel waveguides having two optical states: the bar and cross states. When a directional coupler is in the bar state [Fig. 1(b)], incident light is output from the same side of the waveguide. On the other hand, when a directional coupler is in the cross state 


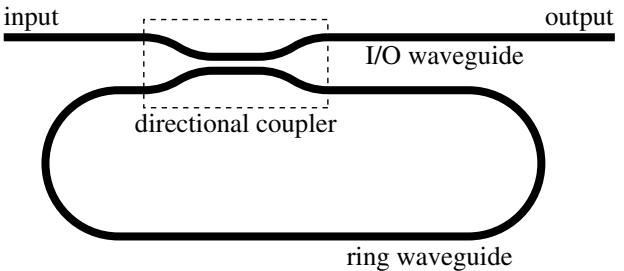

(a)

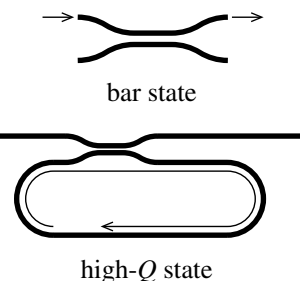

(b)

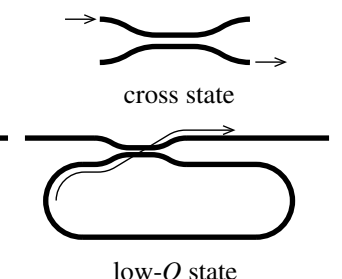

(c)
Fig. 1. (a) Schematic of the ring cavity. It consists of an I/O waveguide and a ring waveguide. These waveguides are optically coupled via a directional coupler. (b) The directional coupler is in the bar state. Light in the ring waveguide is strongly confined (high $Q$ ). (c) The directional coupler is in the cross state. Light in the ring waveguide is immediately extracted (low $Q$ ).

[Fig. 1(c)], all the incident light is transferred to the other side of the coupled waveguide and is output from that side. The length that a coupled waveguide needs to be for light to transfer from one side to the other is called the coupling length, and it depends on the refractive index of the coupled waveguide. Consequently, the coupling length is controlled by modulating the refractive index, enabling switching between the bar and cross states.

The storage state and the R/W state are switched by switching the state of the directional coupler. When the directional coupler is in the bar state, the ring waveguide is optically closed and the light propagating in the ring waveguide is confined in the waveguide. In this case, the $Q$ factor exhibits a high value. On the other hand, when the directional coupler is in the cross state, light incident from the input port propagates into the ring waveguide or light in the ring waveguide is immediately guided to the output port. This weak lightconfinement state has a low $Q$ factor.

For practical use as random-access memory in optical communication, the $Q$ factor of the ring cavity must be controllable over a wide range. This is because a high $Q$ state is required to confine light for a long time, while a low $Q$ state is required for immediate $\mathrm{R} / \mathrm{W}$ of optical signals.

\section{MODULATION OF THE $Q$ FACTOR}

To express the factors that determine the state of the ring cavity, we present an equation that gives the $Q$ factor. The $Q$ factor of the entire ring cavity $\left(Q_{\text {sys }}\right)$ is related to the time decay of the optical energy $U(t)$ in the ring cavity by the following equation:

$$
U(t)=\exp \left(-\frac{\omega t}{Q_{\mathrm{sys}}}\right)
$$

where $\omega$ is the angular frequency of the light. The time decay in the ring waveguide can be divided into two components: a time decay caused by the leak toward the $\mathrm{I} / \mathrm{O}$ waveguide in the directional coupler and that caused by the radiation loss in the ring waveguide. If we explicitly write down these components, we obtain

$$
U(t)=\exp \left(-\frac{\omega t}{Q_{\mathrm{DC}}}\right) \exp \left(-\frac{\omega t}{Q_{\text {ring }}}\right)
$$

where $Q_{\mathrm{DC}}$ is an imaginary $Q$ factor of the entire device without radiation loss in the ring waveguide and $Q_{\text {ring }}$ is a $Q$ factor of a closed (isolated) ring waveguide excluding the coupling to $\mathrm{I} / \mathrm{O}$ waveguide through the directional coupler. From Eqs. (1) and (2), $Q_{\text {sys }}$ is given by

$$
\frac{1}{Q_{\text {sys }}}=\frac{1}{Q_{\text {ring }}}+\frac{1}{Q_{\mathrm{DC}}} .
$$

$Q_{\text {ring }}$ corresponds to $Q_{\text {sys }}$ when there is no directional coupler. This value is mainly determined by the strength of light confinement of the ring waveguide and it is difficult to control. $Q_{\mathrm{DC}}$ corresponds to $Q_{\mathrm{sys}}$ when there is no loss $\left(Q_{\text {ring }}=\infty\right)$ at the ring cavity. $Q_{\mathrm{DC}}$ depends on the coupling rate $\kappa$, which is defined as

$$
\kappa=\frac{I_{\text {cross }}}{I_{\mathrm{bar}}+I_{\text {cross }}},
$$

where $I_{\mathrm{bar}}$ is the output power in the bar direction and $I_{\text {cross }}$ is the output power in the cross direction. When the state of the directional coupler is changed from the bar state to the cross state, $\kappa$ changes from 0 to 1 . If there is no loss at the ring waveguide, the optical energy in the ring cavity decreases by a factor of $1-\kappa$ by propagating through the directional coupler. That is expressed as

$$
1-\kappa=\exp \left(-\frac{2 \pi \omega_{n} T_{0}}{Q_{\mathrm{DC}}}\right),
$$

where $\omega_{n}$ is the normalized frequency, which is equal to $a \omega / 2 \pi c$, and $c$ is the speed of light in vacuum. $T_{0}$ is the time that the light revolves in the ring waveguide one time, which is defined by

$$
T_{0}=\frac{L_{n}}{v_{g}}
$$

where $v_{g}$ is the group velocity of light normalized by $c$ and $L_{n}$ is the entire length of the ring waveguide normalized by the lattice constant $a$. By solving Eq. (ㄷ), we obtain

$$
Q_{\mathrm{DC}}=\frac{2 \pi \omega_{n} L_{n}}{v_{n} \ln \left(\frac{1}{1-\kappa}\right)} .
$$

This shows that $Q_{\mathrm{DC}}$ is easily controlled by varying the coupling $\kappa$ of the directional coupler, so that if $\kappa$ is ideally changed from 0 to $1, Q_{\mathrm{DC}}$ changes from $\infty$ to 0 . Thus, $Q_{\mathrm{sys}}$ is modulated from $Q_{\text {ring }}$ to 0 .

\section{RING CAVITY DESIGN}

\section{A. Control Range of the Conventional Ring Cavity}

A ring cavity that we developed in previous work [14,15] consists of a directional coupler and a ring waveguide, as shown 
in Fig. 1. The directional coupler is a pair of coupled waveguides, and there are three rows of circular air holes between them. The length was set to $80 a$. The ring waveguide has a hexagonal form having six $60^{\circ}$ sharply bent points. The waveguide length is $680 a$. The coupling length has a bar state at around $1348 \mathrm{~nm}$ and a cross state at around $1354 \mathrm{~nm}$. To switch the state of the ring cavity, we need to shift the spectrum by at least $6 \mathrm{~nm}$ (more than $0.4 \%$ in frequency or the refractive index). Instead of the large refractive index modulation, we may extend the length of the directional coupler. As the directional coupler becomes long, the bar and the cross states are switched more frequently in the spectrum, and we can achieve bar-cross switching with a smaller spectral shift. In either case, such modulations have a large energy consumption or modulation time because the refractive index of a large area must be modulated. It is not suitable for the control of fast random-access memory.

Another problem is a considerable loss in the ring waveguide. The $Q_{\text {sys }}$ reported in [14] is $\sim 6000$. The cause of the loss is a radiation loss at the corner of ring waveguide. A $60^{\circ}$ sharply bent waveguide, which is used in the conventional ring cavity, radiates the propagating light, and the radiated light is observed as a bright spot [16]. Because such radiation loss decreases the $Q_{\text {ring }}$, we can hardly achieve a high $Q_{\text {sys }}$ value for a long storage time.

\section{B. Redesign of the Cavity Structure}

To overcome the problem that the directional coupler needs a large spectral shift or a considerably long coupled waveguide, we replace the directional coupler by one having a flat dispersion [17]. The novel directional coupler was fabricated on a GaAs wafer and demonstrates the switching operation [18]. The length of the coupled waveguide is $4.68 \mu \mathrm{m}$. Because the transmittance is drastically changed at around $1368.5 \mathrm{~nm}$, the switching operation is available with a very small spectral shift. We modulated the refractive index by thermal modulation. By a temperature increase of around $57.5^{\circ} \mathrm{C}$, the directional coupler was switched from the bar to the cross state at $1370 \mathrm{~nm}$. Our experimental result also shows that if we achieve that with a conventional (nonflat dispersion) directional coupler, a $234 \mu \mathrm{m}$ long directional coupler is necessary [18].

To reduce the loss in the ring waveguide, we replace the $60^{\circ}$ sharply bent waveguide by a circularly curved waveguide. In a previous paper, we reported that the curved waveguide has lower bending loss than the sharply bent waveguide [16]. Moreover, the backreflection of the propagating light in the bent waveguide can also be reduced. It is effective for removing unexpected Fabry-Perot resonance in the ring waveguide to maintain the shape of stored optical pulses.

The photonic crystal we employed is a two-dimensional photonic-crystal slab. Air-filled holes with a radius of $0.29 a$ are arranged in a triangular lattice (lattice constant $a$ of $390 \mathrm{~nm}$ ) on a $190 \mathrm{~nm}$ thick GaAs slab. Both ends of the directional coupler have adiabatic connectors [19] with lengths of $5 a$ to increase the extinction ratio. The coupled waveguide is finally separated into two single waveguides by a pair of circular waveguides. The ring waveguide is formed by connecting one of the input or output ports with the circular waveguide.
The $Q_{\text {sys }}$ is obtained from the transmission spectrum using

$$
Q_{\mathrm{sys}}^{-1}=\frac{\text { FWHM of resonance-peak }}{\text { peak wavelength }} .
$$

Although the ring cavity needs to be long to store many optical pulses, such a long ring cavity has a very large free spectral range, and it is difficult to measure the $Q$ factor in the frequency domain because neighboring resonance peaks overlap. Therefore, in this study, we used a short length of $520 a$. The ring waveguide consists of straight waveguides and a circularly curved waveguide. The curvature of the curved waveguide was set to be $1.0^{\circ} / a$. To monitor the state of the directional coupler, we also fabricated a single directional coupler having the same structure as that in the ring cavity.

The designed geometry was fabricated by electron-beam lithography on a resist layer over a GaAs epitaxial wafer, and it was transferred to the GaAs epitaxial wafer by ionic dry etching. The sacrificial layer under the top GaAs layer was then undercut by wet etching, producing an air-bridged structure of two-dimensional photonic crystals. Figures 2(a) and 2(b) show scanning electron microscopy (SEM) images of the fabricated ring cavity and its directional coupler, respectively.

\section{DEMONSTRATION OF $Q$ MODULATION}

The $Q$ factor is controlled by varying the refractive index of the directional coupler. In this study, the refractive index was modulated thermally. We installed a Peltier device on the sample stage of the optical measurement system and passed an electric current through it. The temperature of the stage was monitored by a thermocouple thermometer, and transmission spectra were obtained after the temperature had stabilized. Although this method has a slow modulation speed, it does not require any additional optical systems or waveguides for the measurement system or the fabricated device and it is adequate to measure the range over which the $Q$ factor can be controlled.

Transmission spectra were measured using a tunable laser and a photodetector. Light from the tunable laser was input into the I/O waveguide of the ring cavity. The output light from the $\mathrm{I} / \mathrm{O}$ waveguide was picked up by a lensed fiber and guided to the photodetector. The upper side of Fig. 3(a) shows a spectrum of the ring cavity at a temperature of $25.2^{\circ} \mathrm{C}$. The sharp downward peaks separated by intervals in the range 1.0 to $1.5 \mathrm{~nm}$ are resonance peaks of the ring cavity.

The lower side of Fig. 3(a) shows the transmission spectrum of the directional coupler in the bar and cross directions. The ring waveguide and the single directional coupler were fabricated on the same wafer, and they are modulated with an equal temperature. The resonance-peak width in the ring cavity corresponds to the transmittance of the directional coupler; when the directional coupler is in the cross state, resonance peaks of the ring cavity are broad in the corresponding wavelength bands. On the other hand, when the directional coupler is in the bar state, resonance peaks of the ring cavity are sharp. There is the sharpest resonance peak (having maximum $Q$ factor) at $1370.9 \mathrm{~nm}$, while the single directional coupler has a maximum (bar state) transmittance at $1369.4 \mathrm{~nm}$. Because the directional coupler in the ring cavity 


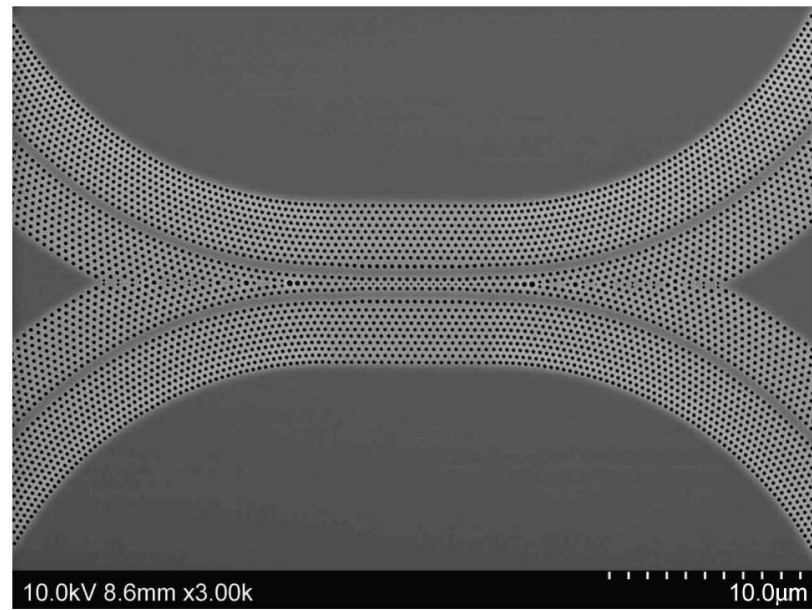

(a)

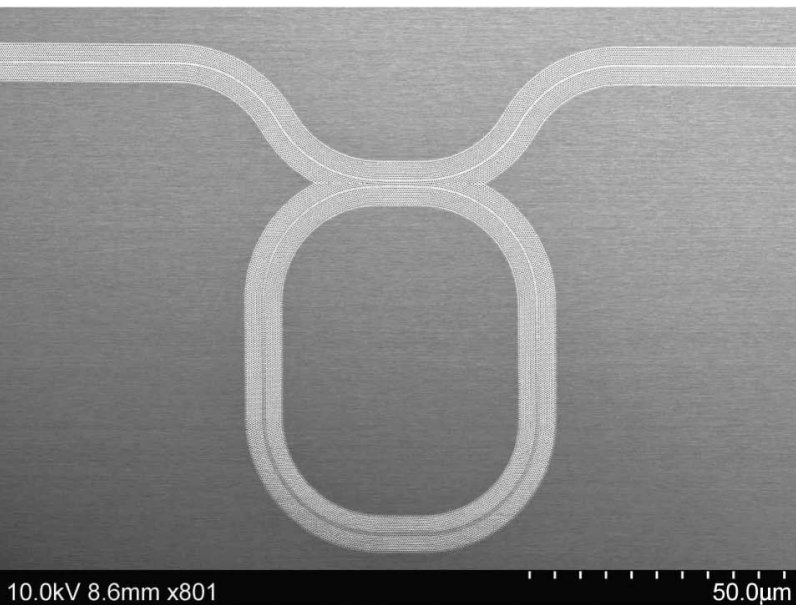

(b)

Fig. 2. Surface SEM images of the (a) entire microring cavity and (b) flat-band directional coupler. The length of the ring waveguide is $520 a$ with a curvature of $1.0^{\circ} / a$. The length of the flat-band directional coupler is $12 a$. Both ends are connected to a $5 a$ long adiabatic connector.

and the single directional coupler has the same structure, their wavelength must be nearly equal. However, there is a relative error of $100 \times(1370.9-1369.4) / 1370.9 \simeq 0.10942[\%]$ between them. The possible reason for the error is a fabrication error of the structure. By measurement of the radii of the air hole in the fabricated device, we found that there is a variation in the radius of around $\pm 4.05 \%$. This error gives a relative error of about $1.82 \%$ in the average refractive index, which is obtained by the refractive index of GaAs (3.4) and air (1.0) and their volume ratio. Because this value is larger than the relative error in the wavelength, it is possibly a reason for the spectral shift. In the spectrum of the fabricated directional coupler, the transmittance is drastically changed at around $1368 \mathrm{~nm}$ (1369.5 $\mathrm{nm}$ for the directional coupler in the ring cavity), showing that the flat band exists at the wavelength. Correspondingly, the resonance-peak width in the ring cavity spectrum also changes drastically at $1369.5 \mathrm{~nm}$. In other words, the $Q$ factor around this wavelength has the potential for large changes by a small spectral shift.

Next, we increased the temperature and measured the change in the $Q$ factor at around $1369.5 \mathrm{~nm}$. The sharpest resonance peak occurs at a wavelength of $1370.9 \mathrm{~nm}$ (indicated by the vertical dashed lines in Fig. 3). Based on Eq. ()), that peak corresponds to the highest $Q$ factor of $1.7 \times 10^{4}$ at $25.2^{\circ} \mathrm{C}$. As the temperature was increased, the refractive index of the sample increased, and the spectrum is redshifted. At temperatures of $50.0^{\circ} \mathrm{C}$ and $68.8^{\circ} \mathrm{C}$ [shown in Figs. 3(b) and 3(c)], the resonance peak appears at $1370.9 \mathrm{~nm}$ again, allowing us to measure the $Q$ factors at these temperatures. At a temperature of $68.8^{\circ} \mathrm{C}$, the resonance peak is broadest because the

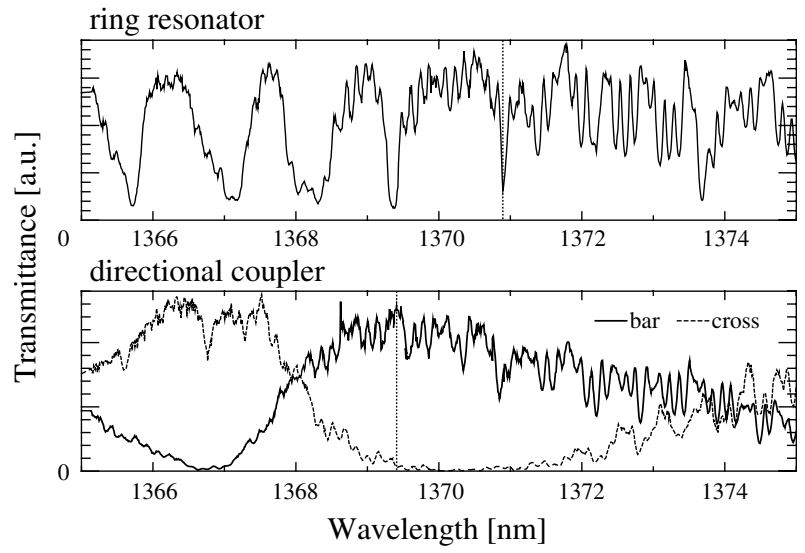

(a)

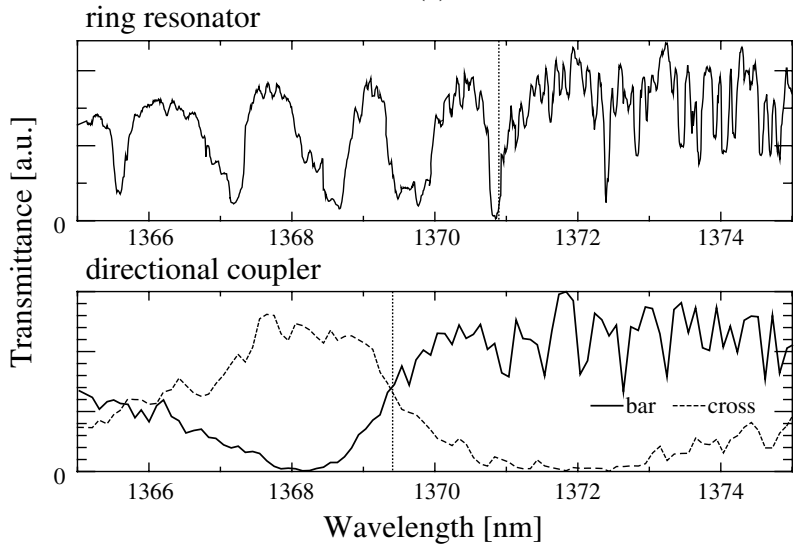

(b)

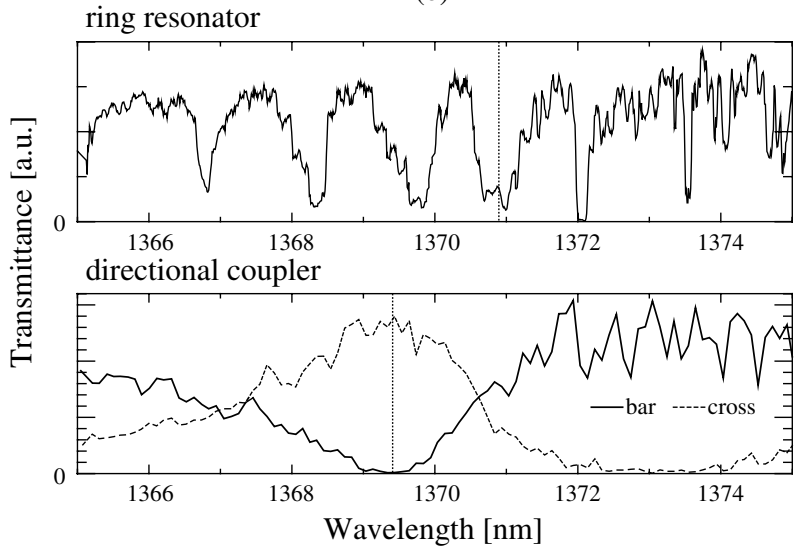

(c)

Fig. 3. Transmission spectra of the ring cavity (left side) and directional coupler (right side) at (a) $25.2^{\circ} \mathrm{C}$, (b) $50.0^{\circ} \mathrm{C}$, and (c) $68.8^{\circ} \mathrm{C}$. The width of the resonance peak depends on the state of the directional coupler; broad peaks corresponds to the cross state, and sharp peaks correspond to the bar state. As the temperature is increased, the spectra are redshifted and the peak at $1370.9 \mathrm{~nm}$ (indicated by vertical dashed lines) becomes sharp. 
directional coupler was switched to the cross state. The $Q$ factor was found to be $1.9 \times 10^{3}$ from the width of the resonance peak. This result reveals that the $Q$ factor can be reduced by a factor of 8.9

\section{DISCUSSION}

In the measurement and analysis described in the previous sections, we observed the $Q$ factor at a fixed wavelength of $1371 \mathrm{~nm}$ and obtained a control range of 1900 to 17,000 . In this experiment, the resonance peaks were shifted as the temperature increased. Indeed, we notice the $Q$ factor of the same resonance mode [the peaks at $1370.9 \mathrm{~nm}$ in Fig. 3(a), $1372.4 \mathrm{~nm}$ in Fig. 3(b), and $1373.6 \mathrm{~nm}$ in Fig. 3(c)] is hardly changed. This is because the temperature of not only the directional coupler but also the ring waveguide is modulated simultaneously. Numerically simulated spectra of a ring cavity are shown in Fig. 4. The calculation is based on the transfer matrix method $[20]$, and the transmittance is given by

$$
\begin{aligned}
I(\omega)= & \frac{1}{4} \mid\left(e^{i k_{e}(\omega) L_{\mathrm{DC}}}+e^{i k_{o}(\omega) L_{\mathrm{DC}}}\right) \\
& +\left.\frac{\left(e^{i k_{e}(\omega) L_{\mathrm{DC}}}-e^{i k_{o}(\omega) L_{\mathrm{DC}}}\right)^{2} e^{\left(-\gamma+i k_{\mathrm{wg}}(\omega) L_{\mathrm{ring}}\right)}}{2-\left(e^{i k_{e}(\omega) L_{\mathrm{DC}}}+e^{i k_{o}(\omega) L_{\mathrm{DC}}}\right) e^{\left(-\gamma+i k_{\mathrm{wg}}(\omega) L_{\mathrm{ring}}\right)}}\right|^{2},
\end{aligned}
$$

where $k_{\mathrm{wg}}, k_{e}$, and $k_{o}$ are wavenumbers of the ring waveguide and the even and odd modes of the directional coupler, respectively. $L_{\mathrm{DC}}$ and $L_{\text {ring }}$ are the lengths of the coupled waveguide in the directional coupler and the ring waveguide. The dispersion relation, $k(\omega)$, is obtained by photonic band

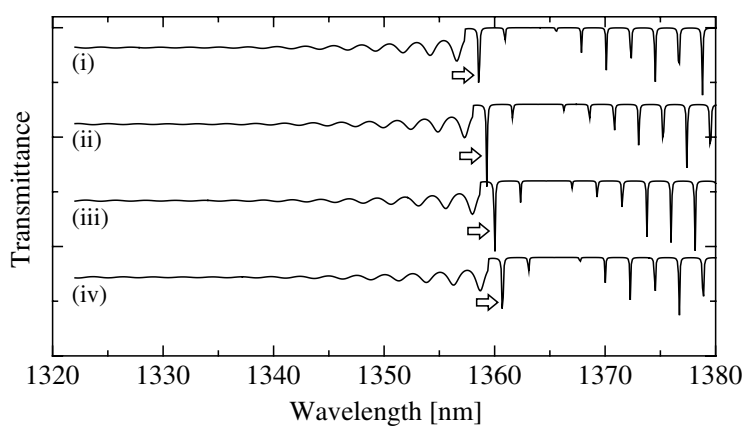

(a)

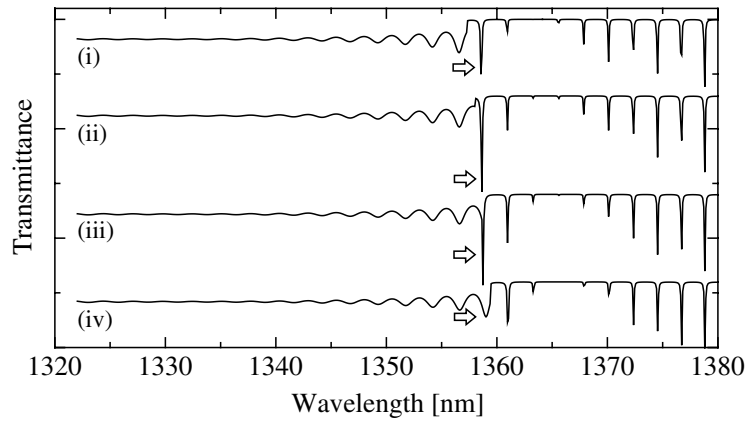

(b)

Fig. 4. Numerically simulated spectra of ring cavities modulated the refractive index of (a) the entire device and (b) only the directional coupler. The spectra are varied from (i) to (iv) by the modulation. The resonance peak of the identical ring resonance mode is indicated by arrows. The widths of the resonance peaks for (a) are hardly varied, while those for (b) are varied, with their peak position fixed. diagrams that were calculated by the three-dimensional finite-difference time-domain method. The $\gamma$, which determines the attenuation of the propagating light in the ring waveguide, was set to 0.1 not depending on the frequency. We assumed that dispersion of the curved waveguide is equal to that of the straight waveguide, according to our previous work in [16]. The temperature modulation is simulated by the frequency shift of the band diagrams. Figure 4(a) is a simulation of when the temperature of both the directional coupler and the ring cavity is increased. As the temperature increased [from (i) to (iv) in Fig. 4(a)], the entire spectrum is redshifted. That behavior corresponds to our experimental result (see Fig. 3). This is because the directional coupler and the ring cavity are on the same substrate, and they are heated with an equal temperature to synchronize the spectral shift. Modulation of the refractive index where the optical pulses are propagating must be avoided so as not to affect the frequency of the optical pulses.

In contrast, Fig. 4(b) is a result of when only the temperature of the directional coupler is modulated. We can see that the resonance-peak width is varied, while those center wavelengths remain unchanged. At this time, the effect of temperature modulation works only for control of the coupling between the I/O waveguide and the ring cavity, and the $Q$ factor of each resonance mode is modulated. Temperature control of only the directional coupler can be achieved by a local heating such as with a microheater or nonlinear materials, instead of the Peltier device.

Even if we modulate only the directional coupler, we expect a nearly equal control range of the $Q$ factor. Figure 5 is the spectrum of a single directional coupler with a flat dispersion and bent waveguide having two circularly $60^{\circ}$ bent parts. The directional coupler has a drastic change in the transmittance at $1368.5 \mathrm{~nm}$. That gives a large change in $Q_{\mathrm{DC}}$. In contrast, the bent waveguide has no observable variation. That indicates that the $Q_{\text {ring }}$ is hardly changed with such a fewnanometer spectral shift in the demonstration in Fig. 3. The control of $Q_{\mathrm{sys}}$ is mainly dependent on the control of $Q_{\mathrm{DC}}$;

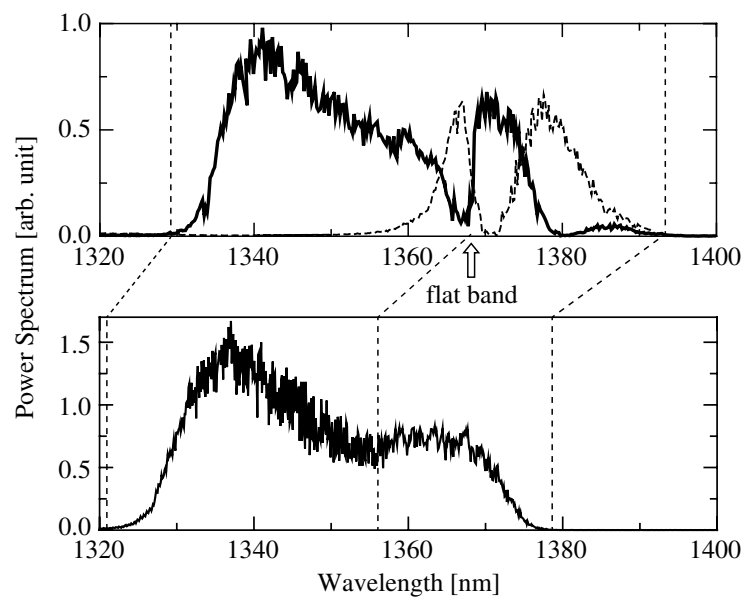

Fig. 5. Spectra of a single directional coupler with a flat band (upper) and a waveguide having two circularly curved $60^{\circ}$ bends (lower). At the wavelength of the flat band $(1368.5 \mathrm{~nm})$, the variation of the transmittance of the bent waveguide is very small. The change of the $Q_{\text {ring }}$ in a spectral shift by around a few nanometers is negligible. We can obtain a nearly equal control range between $1.9 \times 10^{3}$ and $1.7 \times 10^{4}$, even if the refractive index of only the directional coupler is modulated. 
the control range of $Q_{\text {sys }}$ is nearly equal whether the spectrum of the ring waveguide is modulated or not.

We can estimate the storage time from the obtained control range of the $Q$ factor. If we define the storage time $T_{s}$ as the time for the light in the ring cavity to decay to $1 / e$ its initial intensity, $T_{s}$ is given from Eq. (1) by

$$
T_{s}=\frac{Q}{\omega} .
$$

From this equation, the storage time for the maximum $Q$ factor is $12.62 \mathrm{ps}$. On the other hand, the storage time for the minimum $Q$ factor is $1.411 \mathrm{ps}$, which corresponds to the time to extract the light in the ring waveguide to the $\mathrm{I} / \mathrm{O}$ waveguide.

Here, we discuss ways for increasing the control range of the buffering time. The first point is to improve the control range of $Q_{\mathrm{DC}} \cdot Q_{\mathrm{DC}}$ depends on $\kappa$, and if $\kappa$ changes from 0 to $1, Q_{\mathrm{DC}}$ changes from infinity to zero, and $Q_{\mathrm{DC}}$ changes from the maximum $Q_{\text {ring }}$ to 0 . This is the maximum control range of the $Q$ factor. Equation (4) shows that $\kappa$ depends on the extinction ratio. $\kappa$ becomes minimum at the bar state of the directional coupler. In the lower half of Fig. 3(a), the measured directional coupler has a bar state at $1369.4 \mathrm{~nm}$ and a cross state at $1366.5 \mathrm{~nm}$. From the values of the transmittance toward the bar and the cross states and Eq. (4), we can see that $\kappa$ can be controlled over the range $4.93 \times 10^{-3}$ to 0.995. In the measurements, we sometimes observed bright spots at the ends of the directional coupler. These may be caused by narrowing of $\kappa$ owing to cross talk or loss in the separator waveguides at both ends of the coupled waveguide. Thus, we may need to reconsider its structure.

$Q_{\mathrm{DC}}$ also depends on the length of the ring waveguide $L$ and $v_{g} . Q_{\mathrm{DC}}$ can be increased by extending $L$ or reducing $v_{g}$ so that it approaches $Q_{\text {ring. }}$. We obtained the $Q_{\text {ring }}$ of $1.9 \times 10^{4}$ from the measured $Q_{\text {sys }}, \kappa$, and other parameters, and if we can cause $Q_{\text {sys }}$ to approach $Q_{\text {ring, }}$, the storage time will be 14.11 ps. When we use the ring cavity as a buffer circuit for storing many optical pulses, $L$ becomes long. Because the photonic crystal, which contains a ring waveguide, has a low group velocity band, we can reduce $v_{g}$.

If we consider that an optical pulse whose width is a few picoseconds, it is necessary for optical packet buffering to increase the storage time to around nanoseconds. The available maximum $Q$ factor is $Q_{\text {ring }}$, which is determined by the propagation loss in the ring waveguide. The propagation loss mainly depends on the fabrication accuracy, a bending loss at the curved waveguide, and a leak at the directional coupler. The first factor has not been evaluated in our experiment. However, the loss in the waveguide having a similar structure (a triangular-lattice photonic crystal in a GaAs slab) was evaluated in an earlier work [21]. The propagation loss was estimated to be $0.76 \mathrm{~dB} / \mathrm{mm}$. Because this value is for a straight waveguide, the factor of bending loss is not contained. If we assume that the light propagate by the length of $520 a$ (the length of the ring waveguide) with that propagation loss, the transmittance is about 0.97 . If we do not consider the loss at the directional coupler, the $Q_{\text {ring }}$ is equal to the $Q_{\text {sys }}$ that is given from Eq. (1), obtaining $1.7 \times 10^{5}$. In the calculation, the propagation time to circulate the ring waveguide is estimated by using a group velocity in [14]. The corresponding storage time for that $Q$ factor is about $0.13 \mathrm{~ns}$. This value is the maximum storage time when the bending loss is removed. To achieve a storage time of $1 \mathrm{~ns}$, it is necessary to decrease the propagation loss to about $0.10 \mathrm{~dB} / \mathrm{mm}$.

The second factor, loss from the circular waveguide, is known to depend on its curvature radius. In the previous work [16], as the curvature radius of the waveguide increases, the transmittance increased. Because the bending loss must disappear when the curvature radius becomes infinity, the curvature modification is effective for a high $Q$ factor.

The third factor can be solved by extending the length of the ring waveguide. This is effective for not only storing many optical pulses but also to reduce the leak at the directional coupler. As we measured experimentally, the lowest coupling efficiency (value at the bar state) was $4.93 \times 10^{-3}$, not zero. This means that there is a small leak toward the I/O waveguide. The branch waveguides at both ends of the directional coupler slightly leak propagating light because it is difficult to introduce a periodic structure in the branch waveguide, and thus, it is also difficult to achieve high light confinement by the photonic bandgap. As the length is extended, the amount of time to pass through the directional coupler in a unit time, including the branch waveguides, is decreased.

\section{CONCLUSION}

In this study, a microring cavity was designed and fabricated to measure the control range of the $Q$ factor. First, we described that the conventional ring cavity is difficult to be obtained a large control range. That requires a large spectral shift by around $6 \mathrm{~nm}$ in wavelength or a considerably long directional coupler. We also pointed out that the $Q$ factor is limited by weak light confinement of the ring waveguide, owing to the leak at the sharply bent points. As a solution, we replaced the directional coupler and the ring waveguide by a flat-band directional coupler and a low-loss circular-curved waveguide, respectively.

The $Q$ factor was changed by thermal modulation with a Peltier device. At $25.2^{\circ} \mathrm{C}$, the $Q$ factor was $1.7 \times 10^{4}$. As the temperature was increased, the $Q$ factor decreased, and at $68.8^{\circ} \mathrm{C}$, it decreased to $1.9 \times 10^{3}$. This result demonstrates that the storage time can be controlled between 12.62 and $1.411 \mathrm{ps}$. The amount of modulation is around $2.7 \mathrm{~nm}$ in the spectral shift. The approach to heat the entire device modulated the frequency of the resonance mode in the ring cavity. We numerically showed that modulating only the $Q$ factor of each resonance mode is achievable by heating only the directional coupler. We also discussed the experimental result, that the obtained control range is hardly changed when the ring waveguide is not modulated.

Next, we discussed some approaches to enhance the maximum $Q$ factor for a long storage time. The factors for limited storage time are fabrication imperfections, bending loss in the ring waveguide, and leaking at the directional coupler. The achievable storage time was estimated to be $0.13 \mathrm{~ns}$ with the present propagation loss $0.76 \mathrm{~dB} / \mathrm{mm}$. To achieve the storage time of $1 \mathrm{~ns}, 0.10 \mathrm{~dB} / \mathrm{mm}$ is necessary. The bending loss can be decreased by reducing the curvature, and the leak at the directional coupler can be decreased by extending the ring waveguide.

This work has demonstrated dynamical controlling of the $Q$ factor. The next step is to fabricate a system to modulate 
only the directional coupler, not the entire device. That system must realize fast modulation of the $Q$ factor so that the buffering of optical pulses are measured in the time domain.

\section{ACKNOWLEDGMENTS}

This work was supported by the New Energy and Industrial Technology Development Organization (NEDO, no. 08C46215a) and the Japan Society for the Promotion of Science (JSPS) KAKENHI (Grant-in-Aid for JSPS Fellows, no. 2242).

\section{REFERENCES}

1. Y. Akahane, T. Asano, B.-S. Song, and S. Noda, "High- $Q$ photonic nanocavity in a two-dimensional photonic crystal," Nature $\mathbf{4 2 5}$, 944-947 (2003).

2. R. Langenhorst, M. Eiselt, W. Pieper, G. Grosskopf, R. Ludwig, L. Kuller, E. Dietrich, and H. G. Weber, "Fiber loop optical buffer," J. Lightwave Technol. 14, 324-335 (1996).

3. E. Yablonovitch, "Inhibited spontaneous emission in solid-state physics and electronics," Phys. Rev. Lett. 58, 2059-2062 (1987).

4. S. John, "Strong localization of photons in certain disordered dielectric superlattices," Phys. Rev. Lett. 58, 2486-2489 (1987).

5. D. Rafizadeh, J. P. Zhang, S. C. Hagness, A. Taflove, K. A. Stair S. T. Ho, and R. C. Tiberio, "Waveguide-coupled AlGaAs/GaAs microcavity ring and disk resonators with high finesse and $21.6 \mathrm{~nm}$ free spectral range," Opt. Lett. 22, 1244-1246 (1997).

6. B. E. Little, J. S. Foresi, G. Steinmeyer, E. R. Thoen, S. T. Chu, H. A. Haus, E. P. Ippen, L. C. Kimerling, and W. Greene, "Ultra-compact $\mathrm{Si}_{-} \mathrm{SiO}_{2}$ microring resonator optical channel dropping filters," IEEE Photon. Technol. Lett. 10, 549-551 (1998).

7. A. Yariv, "Universal relations for coupling of optical power between microresonators and dielectric waveguides," Electron. Lett. 36, 321-322 (2000).

8. S. T. Chu, B. E. Little, W. Pan, T. Kaneko, S. Sato, and Y. Kokubun, "An eight-channel add-drop filter using vertically coupled microring resonators over a cross grid," IEEE Photon. Technol. Lett. 11, 691-693 (1999).

9. S.-H. Kim, H.-Y. Ryu, H.-G. Park, G.-H. Kim, Y.-S. Choi, Y.-H. Lee, and J.-S. Kim, "Two-dimensional photonic crystal hexagonal waveguide ring laser," Appl. Phys. Lett. 81, 2499-2501 (2002).
10. K. Furuya, N. Yamamoto, Y. Watanabe, and K. Komori, "Novel ring waveguide device in a $2 \mathrm{D}$ photonic crystal slab: transmittance simulated by finite-difference time-domain analysis," Jpn. J. Appl. Phys. 43, 1995-2001 (2004).

11. M. David, F. Monifi, A. Ghaffari, and M. S. Abrishamian, "Heterostructure wavelength division demultiplexers using photonic crystal ring resonators," Opt. Commun. 281, 4028-4032 (2008).

12. S. Kim, J. Cai, J. Jiang, and G. P. Nordin, "New ring resonator configuration using hybrid photonic crystal and conventional waveguide structures," Opt. Express 12, 2356-2364 (2004).

13. D. Goldring, U. Levy, and D. Mendlovic, "Highly dispersive micro-ring resonator based on one dimensional photonic crystal waveguide design and analysis," Opt. Express 15, 3156-3168 (2007).

14. S.-H. Jeong, N. Yamamoto, J. Sugisaka, M. Okano, and K. Komori, "GaAs-based two-dimensional photonic crystal slab ring resonator consisting of a directional coupler and bent waveguides," J. Opt. Soc. Am. B 24, 1951-1959 (2007).

15. S.-H. Jeong, J. Sugisaka, N. Yamamoto, M. Okano, and K. Komori, "Resonant characteristics in a two-dimensional photonic crystal ring resonator with a triangular lattice of air holes," Jpn. J. Appl. Phys. 46, L534-L536 (2007).

16. J. Sugisaka, N. Yamamoto, M. Okano, K. Komori, T. Yatagai, and M. Itoh, "Development of curved two-dimensional photonic crystal waveguides," Opt. Commun. 281, 5788-5792 (2008).

17. N. Yamamoto, T. Ogawa, and K. Komori, "Photonic crystal directional coupler switch with small switching length and wide bandwidth," Opt. Express 14, 1223-1229 (2006).

18. J. Sugisaka, N. Yamamoto, M. Okano, K. Komori, and M. Itoh, "Short photonic-crystal directional coupling optical switch of extended optical bandwidth using flat dispersion," Jpn. J. Appl. Phys. 50, 032201 (2011).

19. N. Yamamoto, Y. Watanabe, and K. Komori, "Design of photonic crystal directional coupler with high extinction ratio and small coupling length,” Jpn. J. Appl. Phys. 44, 2575-2578 (2005).

20. L. F. Stokes, M. Chodorow, and H. J. Shaw, "All-single-mode fiber resonator," Opt. Lett. 7, 288-290 (1982).

21. Y. Sugimoto, Y. Tanaka, N. Ikeda, Y. Nakamura, and K. Asakawa, "Low propagation loss of $0.76 \mathrm{~dB} / \mathrm{mm}$ in GaAs-based singleline-defect two-dimensional photonic crystal slab waveguides up to $1 \mathrm{~cm}$ in length," Opt. Express 12, 1090-1096 (2004). 